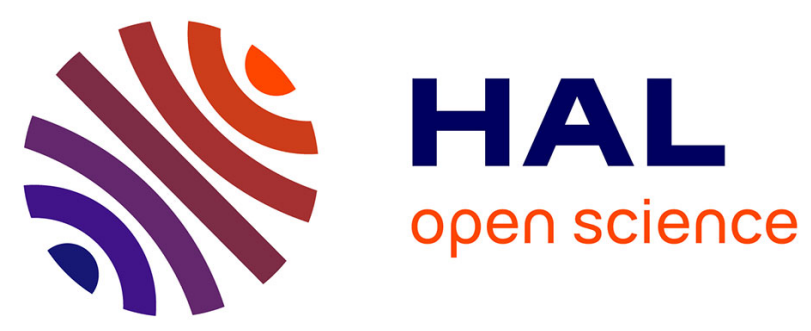

\title{
Numerical efficiency assessment of the lattice Boltzmann model for digital nano-porous rock applications
}

\author{
Aliaksei Pazdniakou, Anne-Julie Tinet, Fabrice Golfier, Kassem Kalo, \\ Stéphane Gaboreau, Patrick Gaire
}

\section{To cite this version:}

Aliaksei Pazdniakou, Anne-Julie Tinet, Fabrice Golfier, Kassem Kalo, Stéphane Gaboreau, et al.. Numerical efficiency assessment of the lattice Boltzmann model for digital nano-porous rock applications. Advances in Water Resources, 2018, 121, pp.44-56. 10.1016/j.advwatres.2018.08.001 . hal-02458489

\section{HAL Id: hal-02458489 \\ https://hal.univ-lorraine.fr/hal-02458489}

Submitted on 16 Oct 2020

HAL is a multi-disciplinary open access archive for the deposit and dissemination of scientific research documents, whether they are published or not. The documents may come from teaching and research institutions in France or abroad, or from public or private research centers.
L'archive ouverte pluridisciplinaire HAL, est destinée au dépôt et à la diffusion de documents scientifiques de niveau recherche, publiés ou non, émanant des établissements d'enseignement et de recherche français ou étrangers, des laboratoires publics ou privés.

\section{(1) (1) $\$$}

Distributed under a Creative Commons Attribution - NonCommercial - NoDerivatives| 4.0 


\title{
Numerical efficiency assessment of the lattice Boltzmann model for digital nano-porous rock applications.
}

\author{
Aliaksei Pazdniakou ${ }^{\mathrm{a}, *}$, Anne-Julie Tinet ${ }^{\mathrm{a}}$, Fabrice Golfier ${ }^{\mathrm{a}}$, Kassem Kalo ${ }^{\mathrm{a}}$, Stephane \\ Gaboreau $^{\mathrm{b}}$, Patrick Gaire ${ }^{\mathrm{a}}$ \\ ${ }^{a}$ Université de Lorraine, CNRS, GeoRessources, F - 54000 Nancy, France \\ ${ }^{b}$ BRGM, Environment and Process Division, F-45060 Orléans, France
}

\begin{abstract}
Study of transport properties of low permeable sedimentary clay formations is of growing interest in exploration of non-conventional resources, and for long term radioactive waste repositories where these formations play the role of a natural shield which prevents the spreading of radioactive materials in the environment. Recent advances in imaging techniques allow to obtain 3D volume of compacted clay sample with pixel size of order of few nano-meters which enables us to explore transport properties of these media by means of numerical simulation. In our study, the Lattice Boltzmann Model (LBM) is applied to simulate fluid flow inside the $3 D$ image of a compacted illite sample to explore its transport properties. The impact of different image segmentation methods applied to treat the raw data on the numerical results is addressed. Because of the nanometric pore size, various physical phenomena may influence fluid flow. In this study, we consider the influence of gas slippage effect (the Klinkenberg effect) on the permeability. The numerically obtained results are compared with experimental data obtained for a reconstructed compacted illite sample. If the original geometry is not strongly modified by the applied image segmentation method, the numerically calculated Klinkenberg factor follows well existing trends and provides predictions close to the experimental values.
\end{abstract}

Keywords: nano-porous media, Lattice Boltzmann Method (LBM), Klinkenberg effect.

\section{Introduction}

The main objective of this study is to improve the understanding of one-phase flow in low permeable porous media by means of numerical simulations performed at the pore scale on $3 D$ images of clay samples. This objective is closely related with the increasing interest in low permeable sedimentary formations which are projected to be used as host site for long term radioactive waste repository [2]. Characterized by low permeability, these media can serve as a natural barrier against radioactive materials. During the

\footnotetext{
* Corresponding author

Email addresses: aliaksei.pazdniakou@univ-lorraine.fr (Aliaksei Pazdniakou), anne-julie.tinet@univ-lorraine.fr (Anne-Julie Tinet), Fabrice.Golfier@ensg.univ-lorraine.fr (Fabrice Golfier)

Preprint submitted to Elsevier 
storage phase, indeed, a certain amount of gas (mostly hydrogen) will be released due to corrosion of iron components of the facility. Increased gas pressure can cause gas migration through the highly water saturated sedimentary material and even affect the geometry of the pore space modifying transport properties of the clay. Therefore, a good understanding of this complex process is of high interest.

Depending on hydrodynamic conditions, various transport mechanisms participating to gas migration can be in action, separately or together [37, 13]. Thus, gas diffusion can be favoured, and the dissolved gas will be transported by advection and diffusion [4]. Conversely, for sufficiently high pressures, gas percolates through the water saturated clay and is transported by continuous visco-capillary flow or small bubble migration. The excess of gas pressure can lead to the dilatation of existing and creation of new pathways $[13,26,15,44]$. Due to nano-metric pore size, however, specific hydrodynamic features need to be considered.

The clayey material has a complex porous structure with the total porosity ranging from $10 \%$ to $40 \%$ (up to $50 \%$ for bentonite), most of which is associated with pores smaller than $100 \mathrm{~nm}[19,37]$. If gas pressure is not sufficiently high, the mean free path of gas molecules $\lambda$ can be significant when compared to the pore size $H$ causing the gas slippage effect. The intensity of gas rarefaction effect is characterized by the Knudsen number Kn

$$
\mathrm{Kn}=\frac{\lambda}{H}
$$

The continuous flow regime corresponds to $\mathrm{Kn}<0.001$; the slip flow is associated with $0.001<\mathrm{Kn}<0.1$; the transitional flow regime corresponds to $0.1<\mathrm{Kn}<10$, and the free molecular flow is related to $\mathrm{Kn}>10$ [40, 24]. For the description of slip flow, the Navier-Stokes equations still can be used together with a slip boundary condition. At the macroscopic scale, the gas slippage effect is manifested in the increased gas permeability $K$, which is known as the Klinkenberg effect [33] and historically expressed as

$$
K=K_{0}\left(1+\frac{b}{P_{m}}\right)
$$

where $K_{0}$ is the absolute permeability, $b$ is the Klinkenberg factor related to the medium properties, and $P_{m}$ is the mean gas pressure.

Recent advances $[19,46]$ in digital rock imaging improved the understanding of the complex hierarchical pore space structure of sedimentary clays. In absence of micrometric fractures, most of the interconnected pores are of nanometric size. The access to the high resolution digital images of real clay samples obtained by FIB-SEM (Focused Ion Beam Scanning Electron Microscope) [19], or at the lower scale by Transmission Electron Microscopy (TEM) if the meso-pores are not connected [16], provides the opportunity to perform numerical simulations of fluid flows, in order to better understand the transport properties of these low permeable porous media. Various numerical methods can be employed to calculate capillary pressure, absolute and relative permeabilities. In our study, we focus on using the lattice Boltzmann method [11]. Originally derived as a generalization of lattice gas automata (LGA), the LBM can be also obtained by discretizing the continuous Boltzmann equation in space, time, and particle velocity space. Unlike the classical Computational Fluid Dynamics (CFD) methods, the LBM describes fluid state by the probabilistic particle distribution function. The model was successfully 
applied to simulate single- and multiphase flow inside the $3 D$ images of porous media $[43,18,7,5]$. The gas slippage effect was introduced later by adopting an appropriate solid-fluid boundary condition. Different formulations have been derived for this purpose based on the pure diffuse reflection [3], its combination with the specular reflection [49] or with the bounce-back boundary condition [11], and a combination of the bounce-back with the specular reflection [47].

Up to now, however, the use of these boundary conditions is limited to simplified and well discretized geometries (e.g., capillary tubes) with the notable exception of the recent application by [54] to 3D digital rocks but without comparison to experimental permeability data. However, challenges still remain to gain reliability in predicting transport properties of such nanoporous materials using Digital Rock Physics (DRP) measurement. First, in spite of the increasing technological advances, it is still unclear whether the spatial resolution available by $3 \mathrm{D}$ microscopic imaging techniques (X-ray tomography, FIB-SEM, BIB-SEM (Broad Ion Beam Scanning Electron Microscope),...) is sufficient to properly capture rock surface features (pore curvature, roughness) that may impact the slippage effect for computer-based simulations. In other words, concerns may be raised whether the filtering process conducted for measuring integrated properties such as permeability possesses sufficient imaging-based microscale information in the presence of slip flow. In adddition, even if the solution of LBM model with slip flow boundary condition has been validated rigorously against fluid flow solution through a parallel-wall uniform fracture or Poiseuille tube flow, applicability to 3D complex pore geometry with non-uniforme pore throats (i.e, with spatially variable Knudsen number) needs to be assessed. The present work aims at addressing these issues.

Our ultimate objective is to explore the capability of the LBM to predict the transport properties of real nano-porous media and confront them to experimental data. An artificial clay material, initially imaged in 3D by [19] will be considered for this purpose. It must be noted that, with the present computational resources, it is not possible to treat the whole image of the sample accounting for all pore scales from micro- to nanometric. Therefore, we are going to work with a selected sub-sample insuring the pore space connectivity. The sensitivity of the numerical methods to the geometry resolution is also investigated. To the best of our knowledge, this is the very first attempt to tackle this complex issue.

\section{Numerical model}

The Navier-Stokes equations describing the flow inside the pore-space can be numerically solved by various computational fluid dynamics (CFD) methods such as finite volume method (FVM) or finite element method (FEM). Recently, non-conventional numerical methods such as LBM received a lot of attention for single- and multi-phase flow modelling in porous media [43, 18]. Indeed, compared to conventional numerical methods, they allow to cope naturally with fluid-fluid interface and complex fluid-solid boundary conditions. Also, various physical phenomena can be relatively easily incorporated in the basic LBM. Finally, possessing a high level of intrinsic parallelism, the LBM can easily profit from parallel computing on CPU and GPU clusters, which is important when dealing with prohibitively large samples [1]. 


\subsection{Lattice Boltzmann Method.}

\subsubsection{The D3Q19 TRT LBM}

The LBM originally appeared as a generalization of LGA in an attempt to overcome some of their difficulties. It was first introduced for a hexagonal lattice with six discrete velocities $(D 2 Q 6)$ in $2 D$ space and a face-centered hypercube $(\mathrm{FHCH})$ lattice in $4 D$ space [38]. Later, it was demonstrated that LBM can be derived from the continuous Boltzmann equation by applying a proper discretization $[27,28]$. The method rapidly extended to various lattices, collision operators, and boundary conditions. Now, it is applied to simulate a wide variety of fluid flow phenomena.

In our study, a regular cubic lattice in $3 D$ space with 19 discrete velocities $(D 3 Q 19)$ is adopted. In LBM, the state of the fluid at each lattice point $\mathbf{r}$ at a given time step $t$ is defined by the particle distribution function $\mathbf{f}=\left(f_{i}(\mathbf{r}, t), i=1, \ldots, Q\right)$, whose components are associated with discrete velocities $\mathbf{c}_{i}$. For a given time moment $t$ each $f_{i}(\mathbf{r}, t)$ defines the probability to find a particle with the discrete velocity $\mathbf{c}_{i}$ at the location $\mathbf{r}$. The 19 discrete velocities for $D 3 Q 19$ are given by

$$
\mathbf{c}_{i}= \begin{cases}(0,0,0), & i=1 \\ ( \pm c, 0,0),(0, \pm c, 0),(0,0, \pm c) & i=2, \ldots, 7 \\ ( \pm c, \pm c, 0),( \pm c, 0, \pm c),(0, \pm c, \pm c) & i=8, \ldots, 19\end{cases}
$$

where the velocity $c=\frac{\delta x}{\delta t}$ is defined as a ratio of the lattice step $\delta x$ and the time step $\delta t$. Both the lattice and the time steps are set to 1 for simplicity. The evolution of the particle distribution function is described by

$$
f_{i}\left(\mathbf{r}+\mathbf{c}_{i} \delta t, t+\delta t\right)=f_{i}(\mathbf{r}, t)-\Omega_{i}\left(\mathbf{f}, \mathbf{f}^{e q}\right)+F_{i} \delta t
$$

where $\Omega_{i}\left(\mathbf{f}, \mathbf{f}^{e q}\right)$ is the collision operator, $F_{i}$ is the discretized body force, and $\mathbf{f}^{e q}$ is the equilibrium distribution. The algorithm can be easily split into two steps, i.e., the calculation of the post-collision distributions in the right part of (4), and the propagation of the post-collision distributions according to the discrete velocities directions.

The collision operator $\Omega_{i}\left(\mathbf{f}, \mathbf{f}^{e q}\right)$ describes the evolution of the particle distribution function due to collision between the particles. The most simple collision operator is the Bhatnagar-Gross-Krook (BGK) collision operator [8] which relaxes the particle distribution function to its equilibrium with a single relaxation time. When used with the bounce-back boundary condition, the well known drawback of this operator is the viscosity dependent location of the boundary. The solution to this problem is the multirelaxation time collision operator (MRT) which relaxes several fluid moments to their equilibrium states with individual relaxation times [17]

$$
\Omega_{i}\left(\mathbf{f}, \mathbf{f}^{e q}\right)=\mathbf{M}^{-1} \cdot \mathbf{S} \cdot \mathbf{M}\left(\mathbf{f}-\mathbf{f}^{e q}\right)
$$

where $\mathbf{M}$ is the transformation matrix, and $\mathbf{S}$ is the diagonal relaxation matrix. The transformation matrix $\mathbf{M}$ projects the particle distribution function onto the moment space giving the following moments [17]

$$
\begin{array}{r}
\mathbf{M} \cdot \mathbf{f}=\left(\rho, e, \varepsilon, J_{x}, q_{x}, J_{y}, q_{y}, J_{z}, q_{z}, 3 p_{x x}, 3 \pi_{x x}, p_{w w}, \pi_{w w},\right. \\
\left.p_{x y}, p_{y z}, p_{x z}, m_{x}, m_{y}, m_{z}\right)
\end{array}
$$


where $\rho$ is the fluid density, and $J_{x}, J_{y}, J_{z}$ are the fluid momentum components; $e$ is related to the kinetic energy and $\varepsilon$ to the kinetic energy square; $q_{x}, q_{y}, q_{z}$ correspond to energy flux, and $3 p_{x x}, p_{w w}, p_{x y}, p_{y z}, p_{x z}$ to the components of the viscous stress tensor; there are also two fourth order moments $3 \pi_{x x}, \pi_{w w}$ and three third order moments $m_{x}, m_{y}, m_{z}$. For the $D 3 Q 19$ model, the $\mathbf{M}$ matrix can be found in [17]. The diagonal matrix $\mathbf{S}$ defines the relaxation rates for different fluid moments [17]

$$
\begin{aligned}
& \mathbf{S}=\operatorname{diag}\left(0, s_{e}, s_{\varepsilon}, 0, s_{q}, 0, s_{q}, 0, s_{q}, s_{\nu}, s_{\pi}, s_{\nu}, s_{\pi}, s_{\nu}, s_{\nu}, s_{\nu},\right. \\
& \left.s_{m}, s_{m}, s_{m}\right)
\end{aligned}
$$

The relaxation rates are positive and must be smaller than 2 for numerical stability. The dimensionless relaxation times are the inverse of the relaxation rates, for example $\tau_{\nu}=\frac{1}{s_{\nu}}$. When using the MRT collision operator, the kinematic $\nu$ and bulk $\zeta$ fluid viscosity can be prescribed separately [17]

$$
\begin{gathered}
\nu=c_{s}^{2}\left(\frac{1}{s_{\nu}}-\frac{1}{2}\right) \delta_{t} \\
\zeta=\left(\frac{5}{9}-c_{s}^{2}\right)\left(\frac{1}{s_{e}}-\frac{1}{2}\right) \delta_{t}
\end{gathered}
$$

where $c_{s}=\frac{c}{\sqrt{3}}$ is the speed of sound. Relaxation times can be tuned in order to improve the precision and numerical stability [17]. The most popular choice is the two relaxation times (TRT) model with two different relaxation times $\tau_{\nu}$ and $\tau_{q}$, which relaxes even and odd order fluid moments with different rates [21]

$$
s_{e}=s_{\varepsilon}=s_{\pi}=s_{\nu}, s_{m}=s_{q}=8 \frac{2-s_{\nu}}{8-s_{\nu}}
$$

The collision operator relaxes the particle distribution function towards its equilibrium state. In LBM, the low Mach number expansion of the Maxwellian distribution is usually used

$$
f_{i}^{e q}(\rho, \mathbf{u})=w_{i} \rho\left[1+\frac{1}{c_{s}^{2}}\left(\mathbf{c}_{i} \cdot \mathbf{u}\right)+\frac{1}{2 c_{s}^{4}}\left(\mathbf{c}_{i} \cdot \mathbf{u}\right)^{2}-\frac{1}{2 c_{s}^{2}}|\mathbf{u}|^{2}\right]
$$

where $\rho$ is the fluid density, $\mathbf{u}$ is the fluid velocity, and $w_{i}$ are the lattice weights. This expansion permits to recover the Navier-Stokes equation at the macroscopic level by using the Chapman-Enskog decomposition. The lattice weights $w_{i}$ in (10) are given by

$$
w_{i}= \begin{cases}\frac{1}{3}, & \left\|\mathbf{c}_{i}\right\|=0 \\ \frac{1}{18}, & \left\|\mathbf{c}_{i}\right\|=1 \\ \frac{1}{36}, & \left\|\mathbf{c}_{i}\right\|=\sqrt{2} .\end{cases}
$$

\subsubsection{Macroscopic variables}

The fluid density $\rho$ and the fluid velocity $\mathbf{u}$ can be obtained as moments of the distribution function

$$
\rho=\sum_{i=1}^{Q} f_{i}=\sum_{i=1}^{Q} f_{i}^{e q}
$$




$$
\rho \mathbf{u}=\sum_{i=1}^{Q} f \mathbf{c}_{i}=\sum_{i=1}^{Q} f_{i}^{e q} \mathbf{c}_{i}
$$

In presence of the body force $\rho \mathbf{a}$, the fluid momentum is redefined as [34]

$$
\rho \mathbf{u}=\sum_{i=1}^{Q} f \mathbf{c}_{i}+\frac{\rho \mathbf{a}}{2} \delta t
$$

in calculation of the nonlinear terms of the equilibrium distribution function as well as the output fluid velocity in order to improve the precision. The pressure is provided by the equation of state of the ideal gas

$$
p=c_{s}^{2} \rho
$$

\subsubsection{Forcing}

The body force $\rho \mathbf{a}$ must be discretized as $\hat{\mathbf{F}}=\left(F_{i}(\mathbf{r}, t), i=1, \ldots, Q\right)$ to be incorporated in LBM. The most common way is to use the following discretization [36]

$$
F_{i}=w_{i} \rho\left[\frac{\mathbf{c}_{i}-\mathbf{u}}{c_{s}^{2}}+\frac{\mathbf{c}_{i} \cdot \mathbf{u}}{c_{s}^{4}}\right] \cdot \mathbf{a}
$$

This discretization allows to use the body force which is not constant in space preserving the Galilean invariance [36]. In this paper following [24], the discretized body force $\hat{\mathbf{F}}$ given by (15) is further relaxed to $\tilde{\mathbf{F}}$ as

$$
\tilde{\mathbf{F}}=\mathbf{M}^{-1} \cdot\left(\mathbf{I}-\frac{1}{2} \mathbf{S}\right) \cdot \mathbf{M} \cdot \hat{\mathbf{F}}
$$

before the incorporation.

\subsubsection{No slip boundary condition}

The interaction between the fluid and solid phase is described by the boundary conditions. The boundary condition must supply the unknown incoming distribution functions for the fluid nodes neighbouring the solid nodes. The most simple and widespread boundary condition is the bounce-back [20]

$$
f_{i}(\mathbf{r}, t+\delta t)=\tilde{f}_{i^{\prime \prime}}(\mathbf{r}, t)
$$

where $\tilde{f}_{i^{\prime \prime}}(\mathbf{r}, t)$ is the post-collision distribution corresponding to the discrete velocity $\mathbf{c}_{i^{\prime \prime}}=-\mathbf{c}_{i}$. According to the bounce back rule, the post-collision distribution is reflected from the solid node in the opposite direction. Combined with the TRT collision operator, this rule provides the second order no-slip boundary condition [21]. The no-slip surface is located in the middle between the fluid and solid lattice nodes. It is possible to change this position by using various interpolation based boundary conditions $[12,21,42,6]$. 


\subsubsection{Slip boundary condition}

When LBM is applied for flow simulations in porous media where $\mathrm{Kn}>0.001$, gas slippage effect can be taken into account by adopting a slip boundary condition. Various approaches permitting to achieve this goal can be employed $[3,47,49,10]$. In our study, the diffuse reflection combined with the bounce-back boundary condition (DBB) is adopted following $[10,11]$

$$
f_{i}(\mathbf{r}, t+\delta t)=r K f_{i}^{e q}\left(\rho_{w}, \mathbf{u}_{w}\right)+(1-r) \tilde{f}_{i^{\prime \prime}}(\mathbf{r}, t), \quad \text { if }\left(\mathbf{c}_{i}-\mathbf{u}_{w}\right) \cdot \mathbf{n}>0
$$

where $\rho_{w}$ is the gas density at the wall, $\mathbf{u}_{w}$ is the wall velocity, $\mathbf{n}$ is the unit wall normal, $0 \leq r \leq 1$ is the combination coefficient, and $K$ is the coefficient defined as

$$
K=\frac{\sum_{\boldsymbol{\xi}_{i^{\prime}} \cdot \mathbf{n}<0}\left|\boldsymbol{\xi}_{i^{\prime}} \cdot \mathbf{n}\right| \tilde{f}_{i^{\prime}}(\mathbf{r}, t)}{\sum_{\boldsymbol{\xi}_{i^{\prime}} \cdot \mathbf{n}>0}\left|\boldsymbol{\xi}_{i^{\prime}} \cdot \mathbf{n}\right| f_{i^{\prime}}^{e q}\left(\rho_{w}, \mathbf{u}_{w}\right)}
$$

where $\boldsymbol{\xi}_{i^{\prime}}=\mathbf{c}_{i^{\prime}}-\mathbf{u}_{w}$, and the incident discrete velocity $\mathbf{c}_{i^{\prime}}$ can be expressed as

$$
\mathbf{c}_{i^{\prime}}=\mathbf{c}_{i}-2\left(\mathbf{c}_{i} \cdot \mathbf{n}\right) \mathbf{n}
$$

According to [11], this boundary condition is more adapted to be used in porous media since it requires only local information.

For simulation of the gas slip flow, the relaxation time $\tau_{\nu}$ must be related to the Knudsen number. The Knudsen number can be related to the viscosity as [22]

$$
\mathrm{Kn}=\sqrt{\frac{\pi}{2}} \frac{\nu}{c_{s} H}
$$

Then, using (8a), $\tau_{\nu}$ can be expressed as [22]

$$
\tau_{\nu}=\frac{1}{2}+\sqrt{\frac{6}{\pi}} \frac{H}{\delta x} \mathrm{Kn}
$$

The relaxation time $\tau_{q}$ and the combination coefficient $r$ can be tuned to comply with a prescribed slip boundary condition. Usually [11], this is done by considering the stationary Poiseuille flow between the flat walls $y=0$ and $y=H$ driven by the body force $\rho\left(a_{x}, 0,0\right)$ with a slip boundary condition prescribed at the walls. In this case, the analytical solution for the parabolic velocity profile $u_{y}$ is given by

$$
\frac{u_{y}}{u_{c}}=4 \frac{y}{H}\left(1-\frac{y}{H}\right)+\frac{u_{s}}{u_{c}}
$$

where $u_{c}=\frac{a_{x} H^{2}}{8 \nu}$ is the velocity at the center $y=\frac{H}{2}$ for the no-slip boundary, and $u_{s}$ is the slip velocity at the wall. The second order slip boundary condition can be written as $[25]$

$$
u_{s}=\left.C_{1} \lambda \frac{\partial u}{\partial y}\right|_{w a l l}-\left.C_{2} \lambda^{2} \frac{\partial^{2} u}{\partial y^{2}}\right|_{\text {wall }}
$$

where $C_{1}$ and $C_{2}$ are the coefficients describing the gas-wall interaction and can be supplied by the kinetic theory $[25,48]$ or experiments. The usual first order slip boundary 
condition corresponds to $C_{2}=0$. Following [25], $C_{1}=1.11$ and $C_{2}=0.61$ values are adopted in this paper due to a good agreement with the solution of the linearized Boltzmann equation up to $\mathrm{Kn} \approx 0.4$. Using (23), the condition (24) can be simplified as

$$
\frac{u_{s}}{u_{c}}=4 C_{1} \mathrm{Kn}+8 C_{2} \mathrm{Kn}^{2}
$$

On the other hand, by using the relation between the velocities at two fluid LBM nodes next to the wall and $(23), u_{s}$ can be expressed as $[23,11]$

$$
\frac{u_{s}}{u_{c}}=\frac{4 r}{2-r} \sqrt{\frac{6}{\pi}} \mathrm{Kn}+\frac{32\left(\tau_{\nu}-\frac{1}{2}\right)\left(\tau_{q}-\frac{1}{2}\right)-6}{\pi\left(\tau_{\nu}-\frac{1}{2}\right)^{2}} \mathrm{Kn}^{2}
$$

Comparing (25) and(26), the combination coefficient $r$ and the relaxation time $\tau_{q}$ can be related to the $C_{1}$ and $C_{2}$ parameters and relaxation time $\tau_{\nu}$ as [11]

$$
\begin{gathered}
r=\frac{2 C_{1}}{\sqrt{\frac{6}{\pi}}+C_{1}} \\
\tau_{q}=\frac{1}{2}+\frac{3+\pi\left(2 \tau_{\nu}-1\right)^{2} C_{2}}{8\left(2 \tau_{\nu}-1\right)}
\end{gathered}
$$

\section{Validation tests}

Before tackling the complex issue of comparing with real experimental data, preliminary validations are to be conducted to assess the solution accuracy. The effect of the mesh refinement and the slip boundary condition are investigated for different geometries. In the following, all the values are given in lattice units unless the units are stated explicitly.

\subsection{Poiseuille flow in a horizontal or inclined channel}

The simplest way to validate the code is to consider a slip flow between two horizontal parallel walls, $y=0$ and $y=H$, driven by a body force $\mathbf{F}=\left(F_{x}, 0,0\right)$. The analytical solution in this case is given by (23). In order to estimate the influence of discretization, simulations are performed for various values of $H$ ranging from 5 to 41 . The (DBB) boundary condition (18) is applied. The obtained dimensionless velocity profiles $\frac{u_{y}}{u_{c}}$ for $\mathrm{Kn}=0.1$ are displayed in Fig. 1. If the bounce-back boundary condition (17) combined with Eq. (9) results in exact velocity profile for the classical Poiseuille solution (i.e., with no-slip condition) [12], in contrast, a small but non-zero error persists in the presence of slippage at the solid wall. The relative error for the velocity at the center of the channel decreases from $0.018 \%$ for $H=5$ to $2.93 \cdot 10^{-4} \%$ for $H=41$. Clearly, the numerical solution is very close to the analytical profile even for $H=5$. The residual error is probably due to the spurious higher-order terms in the non-equilibrium distribution $f_{i}$. Note that a regularization procedure was proposed in [48] to improve the solution accuracy but numerical tests that we performed have shown that it delivers larger relative errors, at least for the range of Knudsen numbers of interest $(\mathrm{Kn}<1)$. These observations suggest that the introduction of slip flow boundary do not dramatically impact the LBM model sensitivity to computational mesh when the exact geometry is known. 


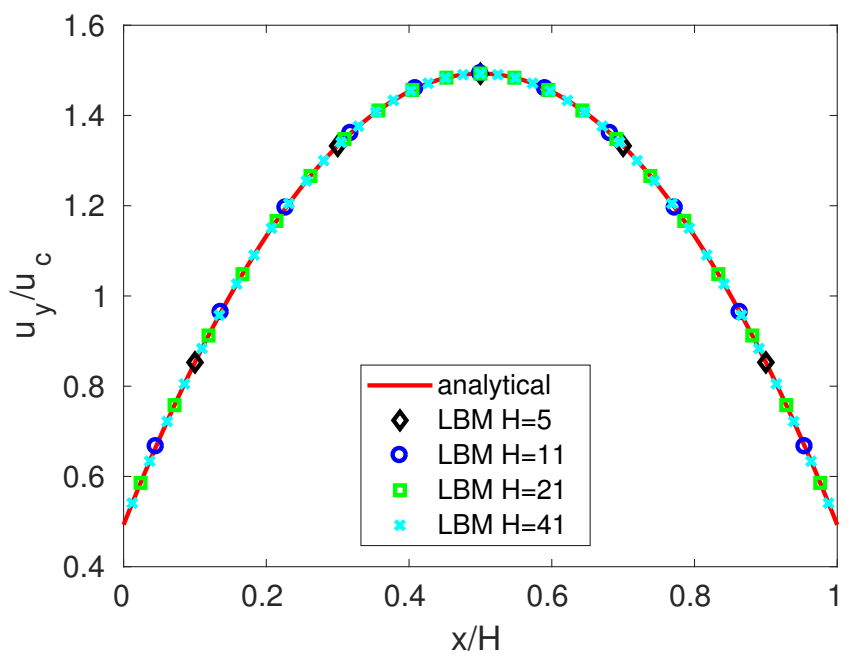

Figure 1: Dimensionless velocity profiles for the Poiseuille flow between horizontal parallel walls $(\mathrm{Kn}=$ $0.1)$.

In a second more complicated case, the channel is inclined now with $45^{\circ}$ angle to the main lattice directions, i.e., it is aligned with $y=x$ as displayed in Fig. 2(a). In practice, when the bounce-back (17) or the DBB (18) boundary condition is applied, the actual geometry corresponds to a staircase shape with the physical solid-fluid interface lying in the middle between the fluid and solid nodes. The LBM cartesian mesh generates a pseudo-roughness similar to the one caused by the voxel-based representation in 3D digitized images. From this test case, we investigate the influence of this loss of geometric information on the solution accuracy with and without slippage. First, the numerical solution of the fluid flow simulated in such geometry applying the bounce-back boundary condition can be compared with the analytical solution for the Poiseuille flow [12] with $H$ defined as in Fig. 2(a). A very good agreement is observed as shown in Fig. 2(b), however, in contrast to the horizontal channel, the numerical solution is no longer exact [12]. From [12], the relative error in $K_{B B}$ when compared to the exact value $K_{0}$ due to this constant shift in the velocity profile can be estimated as $-\frac{1}{4 H^{2}}$. The relative error gets significantly larger when the DBB boundary condition is used to simulate the slip flow in this configuration, as it can be observed from Fig. 3(a) where the numerical solution for $H=\frac{15}{\sqrt{2}}, H=\frac{31}{\sqrt{2}}$, and $H=\frac{63}{\sqrt{2}}$ is compared to the analytical solution. It also follows, that the relative error $\approx 11.45 \%$ has little variation with discretization, while it strongly depends on $\mathrm{Kn}$ as it can be observed in Fig. 3(b) where the dimensionless permeability of the $45^{\circ}$ inclined channel $\left(H=\frac{15}{\sqrt{2}}\right)$ is compared with the analytical solution

$$
K_{\text {theo }}=K_{0}\left(1+6 C_{1} \mathrm{Kn}+12 C_{2} \mathrm{Kn}^{2}\right)
$$

where $K_{0}=\frac{H^{2}}{12}$ is the absolute permeability of the channel. The numerically calculated permeabilities do not follow the parabolic law (28) but can be very well fitted with linear function

$$
K_{L B M}=K_{D B B}(0.95444+5.46444 * \mathrm{Kn})
$$




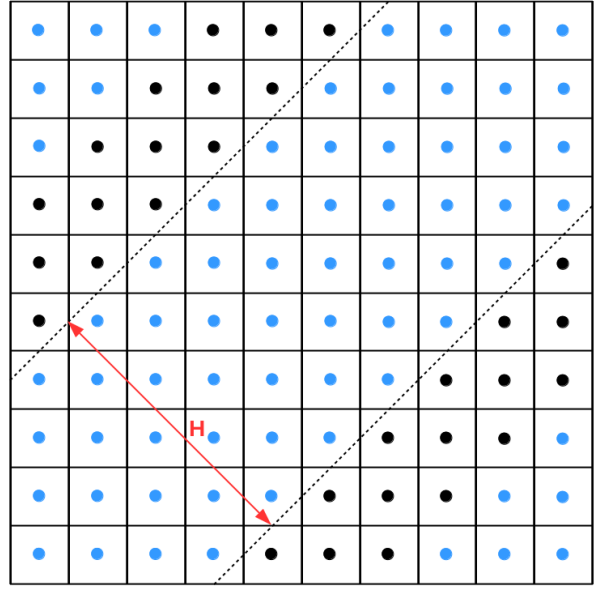

(a)

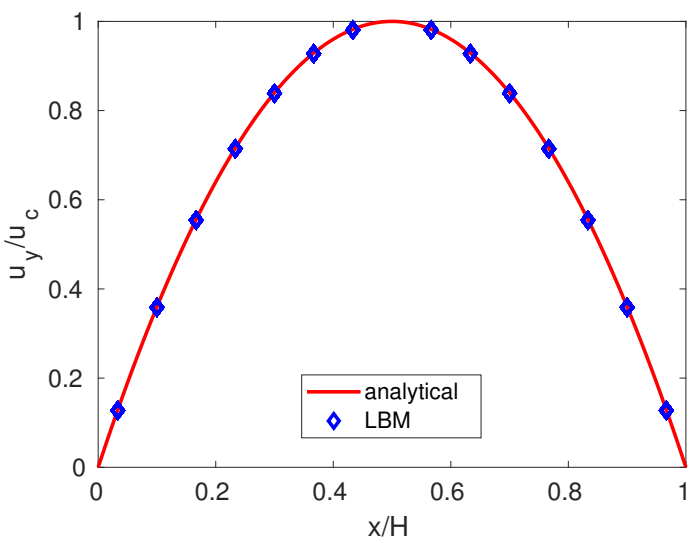

(b)

Figure 2: (a) Schematic view of the unit cell with a $45^{\circ}$ inclined channel. (b) Dimensionless velocity profile for the Poiseuille flow inside the $45^{\circ}$ inclined channel with the bounce-back boundary condition applied at the solid-fluid interface $\left(H=\frac{15}{\sqrt{2}}\right)$.

Originally, the relation (26) between the normalized slip velocity $\frac{u_{s}}{u_{c}}$ and LBM parameters is obtained for the solid-fluid boundary located in the middle between the solid and fluid nodes. The condition is well defined, if we consider that the staircase geometry of the inclined channel is the true geometry. However, if we consider that this geometry corresponds to the inclined channel defined by the dashed lines in Fig. 2(a), the boundary condition (26) is not well defined for some LBM discrete velocities, i.e., the coefficients for the $\mathrm{Kn}$ and $\mathrm{Kn}^{2}$ should contain extra terms due to the actual position of the solid-fluid interface as can be found in [51]. Thus, considering the normalized slip velocity $\frac{u_{s}}{u_{c}}$, the source and the magnitude of the error is exactly the same for all the channels inclined at the same angle regardless the distance between the walls. Therefore, the error does not effectively decrease as $H$ increases, and, as demonstrated in Fig. 3(b), the error grows with Kn.

Obviously, the sensitivity of the solution to the wall roughness increases as the slip effects prevail. This finding suggests that a very careful attention is required for capturing the geometric features of nanoporous materials when Kn becomes large. Another important observation is that the permeability $K_{L B M}$ does not converge to $K_{B B}$ (the permeability calculated using the bounce-back boundary condition) when $\mathrm{Kn} \rightarrow 0$ but to a slightly smaller value $K_{D B B}$, i.e., the error introduced by the DBB condition does not disappear completely when $\mathrm{Kn} \rightarrow 0$.

It was mentioned that this deviation from the analytical solution can be effectively corrected by using the interpolated DBB boundary condition proposed by [51] which places the solid-fluid interface exactly at the positions indicated by the dashed lines in Fig. 2(a) thus eliminating the artificial walls roughness. However, this option is not available for the geometries based on the FIB-SEM images which contain some degree of uncertainty about the actual location of the pore walls and where the solid-fluid interface 


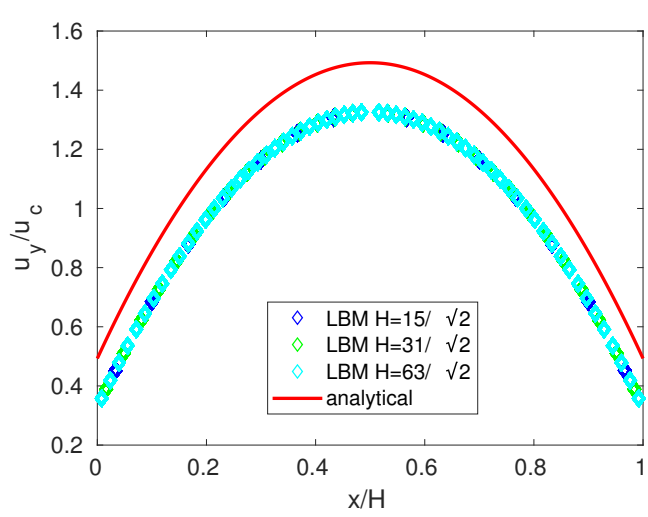

(a)

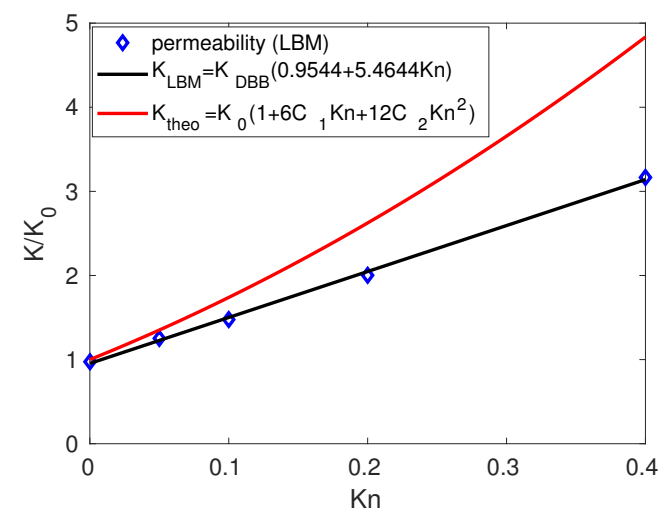

(b)

Figure 3: (a) Dimensionless velocity profiles for Poiseuille flow inside the $45^{\circ}$ inclined channel with the DBB boundary condition applied at the solid-fluid interface $(\mathrm{Kn}=0.1)$. (b) Dimensionless permeability of the $45^{\circ}$ inclined channel as function of $\operatorname{Kn}\left(H=\frac{15}{\sqrt{2}}\right)$.

is described by the border of the cubic voxels. As a consequence, we will not consider this numerical improvement in our model hereafter but keeping in mind this source of error for further comparison with experimental measurement.

\subsection{Permeability of square arrays of circular cylinders}

A more sophisticated geometry which can serve as an approximation of a porous medium is a two-dimensional square array of circular cylinders. The unit cell displayed in Fig. 4 represents the approximation of the solid cylinder inside the square region filled with fluid. A spatially periodic boundary condition is applied at the unit cell sides, and the flow inside the cell is driven by the body force $\mathbf{F}=\left(F_{x}, 0,0\right)$ which acts as a macroscopic pressure gradient $\nabla P$. When stationary flow is obtained, the permeability can be found from the Darcy law

$$
<\mathbf{v}>=-\frac{K}{\mu} \nabla P
$$

In [11], the authors have performed high resolution simulations to study gas flow with Klinkenberg effect through a similar square array of solid cylinders. Their numerical domain contains multiple contiguous unit cells like the one displayed in Fig. 4 with pressure difference imposed at the opposite sides of the domain while spatially periodic boundary conditions are applied at the lateral sides. Several $(n=4[11])$ replicated unit cells inside the domain are necessary in order to obtain grid independent results. When equilibrium is reached, the gas permeability can be calculated as [11]

$$
K=\frac{2 L \mu<U_{o}>p_{o}}{p_{i}^{2}-p_{o}^{2}}
$$

where $L=l \cdot n$ is the length of the domain, $\left\langle U_{o}\right\rangle$ is the averaged velocity at the outlet, $p_{i}$ is the gas pressure at the inlet, and $p_{o}$ is the pressure at the outlet. 


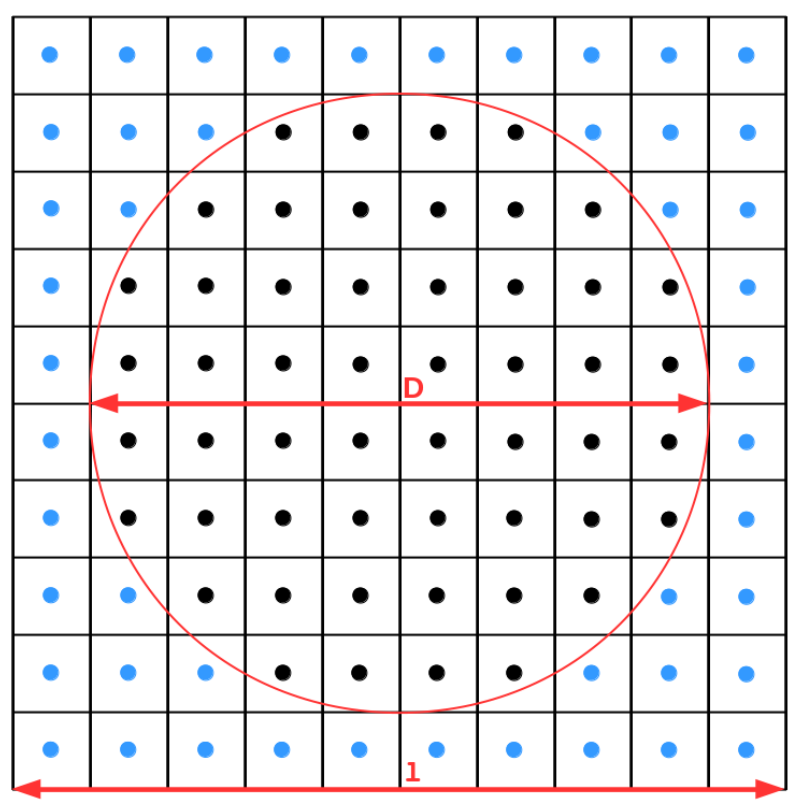

Figure 4: Schematic view of the unit cell of square array of circular cylinders.

If the fluid density variation inside the domain is small, the permeability values calculated from Eqs. (30) and (31) are very close to each other. Thus, the use of the small unit cell (Fig. 4) with spatially periodic boundary conditions and body force as a macroscopic pressure gradient allows us to decrease the computational load without loss of precision. From these simulations, we study the mesh resolution required for predicting with accuracy the permeability of this simplified porous medium. The simulations conducted by Chai et al. [11] are used as the reference solution. The simulations are performed with the unit cell size $l=400$, and the cylinder diameter $D=361$. In order to estimate the influence of the discretization, smaller unit cells are also considered with $l$ as small as $l=25$ while preserving the $\frac{D}{l}$ ratio. The body force $\mathbf{F}=\left(10^{-7}, 0,0\right)$ is small enough, so that the fluid density variations are negligible. Following [11], the Knudsen number is defined using $D_{\text {pore }}$ as $H$

$$
H=D_{\text {pore }}=\frac{\varepsilon}{1-\varepsilon} D
$$

where $\varepsilon$ is the porosity of the unit cell. The obtained permeabilities for various Kn values are compared with the fitting curve $K=K_{0}\left(1+22.52 \mathrm{Kn}+26.87 \mathrm{Kn}^{2}\right)$ from [11] in Fig. $5(\mathrm{a})$, and the corresponding relative deviations from this curve are displayed in Fig. 5(b).

As expected, the deviation from the [11] fitting curve grows with degradation of discretization. It can be concluded that at least 6 lattice nodes (i.e., $l=60$ ) spanning the pore throat are necessary in order to have the permeability value within the $10 \%$ accuracy range, thus demonstrating that the method is sufficiently robust and it can be used for simulations in coarsely discretized geometries with a reasonable precision. 


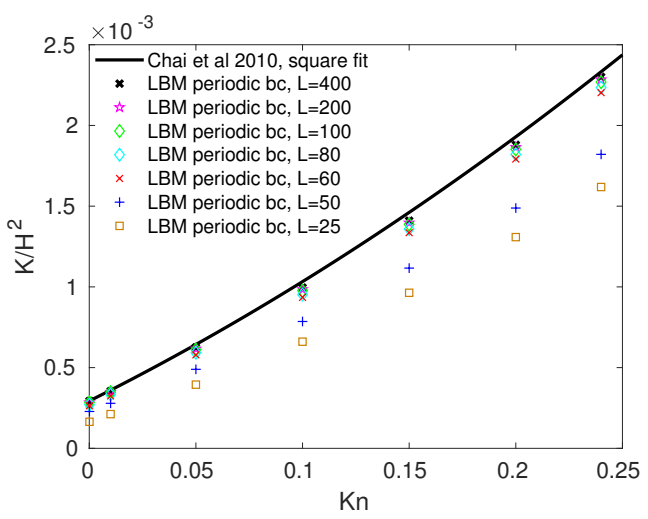

(a)

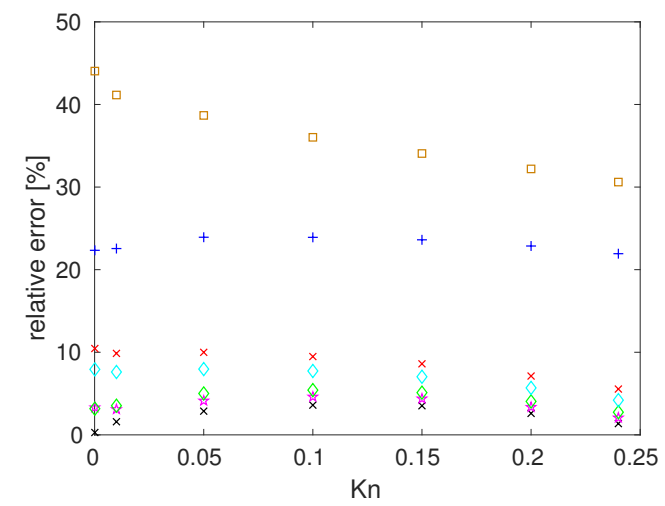

(b)

Figure 5: (a) Permeability of the cubic array of circular cylinders as function of Kn for various discretizations. (b) Permeability deviation from fitting curve provided by [11] for various discretizations.

\section{Application to illite sample}

The availability of extremely fine images of compacted illite sample obtained by FIBSEM [19] provides us the possibility to study its transport properties by means of numerical simulation. The obtained results can be thereafter confronted with experimental results obtained for the compacted illite sample of the same origin.

\subsection{Experimental results}

The images provided by [19] correspond to the illite extracted from the Le Puy en Velay basin. The powdered illite was purified and compacted to reach the dry density of $1.7 \mathrm{~g} / \mathrm{cm}^{3}$. The composition of the illite and the preparation processes can be found in [19]. However, since the transport properties were not measured by [19], a centimetre size sample was recreated from the same basic material, and the permeabilities to water and gas were experimentally measured. The measurement of permeability to gas was performed in an oedometer cell under a constant normal load of $89 \mathrm{MPa}$ to ensure a dry density of $1.7 \mathrm{~g} / \mathrm{cm}^{3}$. Nitrogen is injected at constant pressure and the outflow at equilibrium is measured using a soap bubble flowmeter. The permeability is then calculated by using (31). The permeability to water is also measured by maintaining a normal load which ensures a dry density of $1.7 \mathrm{~g} / \mathrm{cm}^{3}$. First, the sample is saturated by applying water pressure on both sides with a gradient of $0.2 \mathrm{MPa}$, until the equilibrium is obtained. Then, the water is injected at constant pressure and the outflow is measured in stationary conditions to obtain the permeability by using (30).

The measured water permeability is $K_{\mathrm{H}_{2} \mathrm{O}}=2.14 \cdot 10^{-20} \mathrm{~m}^{2}$. The gas permeability values measured for the entry gas pressure ranging from $1.738 \mathrm{MPa}$ to $12.298 \mathrm{MPa}$ are displayed in Fig.6(a) as function of $\frac{1}{P_{m}}$. Using the least squares fitting, the experimental data can be approximated by (2) with $K_{0}=4.7952 \cdot 10^{-20} \mathrm{~m}^{2}$ and $b=7.5748 \cdot 10^{6}$ $\mathrm{Pa}$. The red point in Fig.6(a) corresponding to the $K$ value measured at $p_{i}=12.298$ MPa was excluded from the fitting set (the blue points) since at this high pressure, the 


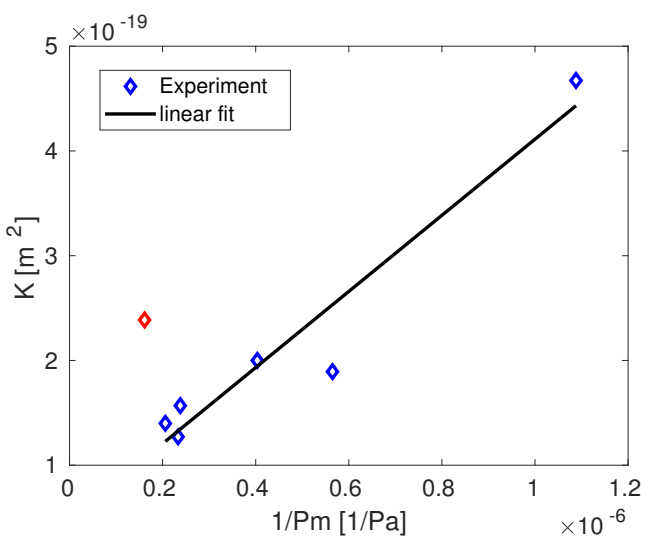

(a)

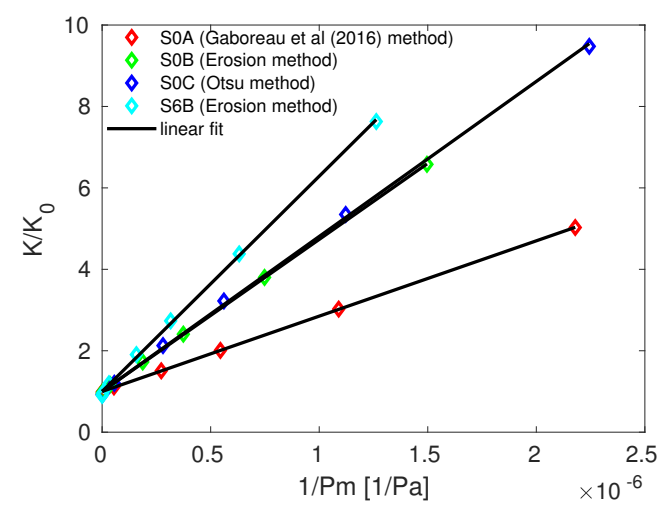

(b)

Figure 6: (a) Experimentally measured permeabilities of the compacted illite sample versus the inverse of the mean gas pressure. (b) Dimensionless numerically calculated permeabilities of S0x and S6B sub-samples versus the inverse of the mean gas pressure. Solid lines are linear fits by (2).

permeability starts to grow significantly which can be attributed to the modification of the initial pore space geometry. Therefore, no measurements at higher pressures where performed. The obtained $K_{0}$ value is 2.24 times higher than the $K_{H_{2} O}$ value. The differences can be attributed to the limitations of the experimental equipment and the employed measurement technique as well as uncertainties of the measured values. Another possibility is the specific interaction of water molecules with solid walls inside the nano-pores of clayey material. Indeed, according to [39], the absolute permeability for clay-rich rocks estimated from the Klinkenberg correlation (2) is routinely $2-3$ times higher than the permeability measured to a liquid.

Another series of gas permeability measurements on a different compacted illite sample was performed for relatively low entry gas pressures $\left(p_{i}<1.6 \mathrm{MPa}\right)$. A non-linear behaviour of the measured permeability which does not follow the linear correlation (2) is observed. A similar behaviour was observed for COx argillite sample in [9] when $p_{i}<0.86 \mathrm{MPa}$.

\subsection{Numerical results}

\subsubsection{Image sampling}

Image segmentation was performed by Gaboreau et al [19] using different methods: an advanced method that allows the recovery of porosity and pore distribution (see Fig.7(a)), an erosion algorithm (see Fig.7(b)), and the Otsu algorithm (see Fig.7(c)). The Otsu algorithm is an often used segmentation method, in the case of the illite sample, it allows a good determination of the largest pores, essential to fluid flow [19]. The erosion method favors pore connectivity and lessens isolated pores. The method favored by Gaboreau et al [19], based on watersheding, shows the best fit in regards to total porosity and pore size distribution. It should be noted, however, that the fitting between image data and macroscopic measurements of porosity and pore size distribution is only fully valid if the image is representative of the sample. Gaboreau et al [19] calculate the REV 
(Representative Elementary Volume) based on the analysis of 2D grayscale images and obtains a REV of $1000-1200 \mathrm{~nm}$. The already segmented images are $1096 \times 1095$ pixels large and constitute three stacks of 180 images each, meaning that the REV, as calculated by Gaboreau et al [19], is not reached in the third direction. The pixel size is $5 \times 5 \mathrm{~nm}$. The distance between the consecutive images is also $5 \mathrm{~nm}$ [19], therefore, the voxel size is $5 \times 5 \times 5 \mathrm{~nm}$. The porosity $\varepsilon=0.2928$ of the Fig.7(a) sample is larger than the porosity $\varepsilon=0.1312$ of the Fig.7(b)) sample which is very close to the porosity $\varepsilon=0.1313$ of the Fig.7(c)) sample. The availability of three image stacks allows us to study the influence of the segmentation techniques on the numerically calculated transport properties.

In order to assess the role of segmentation in regards to permeability, for simulation tests, three sub-samples, hereinafter referred to as S0A, S0B, and S0C, of the same size are extracted from the three image stacks at the same location indicated by the red rectangular in Figs.7(a), (b), and (c), respectively. The sub-samples size is $101 \times 101 \times 102$ voxels in order to avoid high computational costs. Several solid voxels suspended inside the pore space as well as disconnected pores were removed from the sub-samples making the pore space fully connected. The total $\varepsilon_{t}$ and connected $\varepsilon_{c}$ porosities are summarized in Tab.1. All three sub-samples are percolating and can be used for fluid flow simulation.

\begin{tabular}{|l|c|c|c|c|}
\hline & SOA & SOB & SOC & S6B \\
\hline$\varepsilon_{t}$ & 0.2963 & 0.1197 & 0.07619 & 0.1099 \\
$\varepsilon_{c}$ & 0.2926 & 0.1132 & 0.04728 & 0.1024 \\
\hline$<H_{l}>_{x}, \mathrm{~nm}$ & 39.1051 & 30.3775 & 61.6270 & 31.2943 \\
$<H_{l}>_{y}, \mathrm{~nm}$ & 53.1879 & 42.1561 & 74.8762 & 41.7924 \\
$<H_{l}>_{z}, \mathrm{~nm}$ & 88.2249 & 67.4073 & 162.006 & 42.0770 \\
$<H_{l}>, \mathrm{nm}$ & 53.4750 & 40.1369 & 79.1668 & 36.3871 \\
\hline$K_{B B}(x), \mathrm{m}^{2}$ & $1.0357 \cdot 10^{-18}$ & 0 & $1.2625 \cdot 10^{-20}$ & $2.9750 \cdot 10^{-20}$ \\
$K_{B B}(y), \mathrm{m}^{2}$ & $1.3822 \cdot 10^{-18}$ & $4.7875 \cdot 10^{-20}$ & 0 & $9.2475 \cdot 10^{-20}$ \\
$K_{B B}(z), \mathrm{m}^{2}$ & $2.5568 \cdot 10^{-18}$ & $1.7428 \cdot 10^{-19}$ & $1.3475 \cdot 10^{-20}$ & $5.3850 \cdot 10^{-20}$ \\
\hline$K_{0}, \mathrm{~m}^{2}$ & $2.3556 \cdot 10^{-18}$ & $1.4875 \cdot 10^{-19}$ & $1.3030 \cdot 10^{-20}$ & $4.7242 \cdot 10^{-20}$ \\
$b, \mathrm{MPa}$ & 1.8250 & 3.6629 & 3.4970 & 5.0414 \\
\hline \multicolumn{4}{r|}{} \\
\hline
\end{tabular}

Table 1: Geometrical and transport characteristics of SOx, S6B sub-samples.

Only S0C sub-sample demonstrates significant difference between the connected and total porosities due to lower connectivity. Before the simulation, all the sub-samples are mirrored along the chosen axis in order to assure connections between the pores at the opposite sides of the sub-sample when the spatially periodic boundary condition is applied.

Considering the simulation size restriction, it can be observed that the simulated volumes are below the REV, calculated from grayscale images. On top of that, REV actually depends on the process studied, with REV for permeability being most likely larger than porosity REV [53] or grayscale images REV. In order to compensate for these limitations, a statistical study on different sub-samples is necessary. Spatial variations of geometrical and transport properties are estimated by considering 9 additional subsamples of the same size $(101 \times 101 \times 102$ voxels $)$ extracted from each of the three original image stacks at locations displayed in Fig.8(a). The sub-samples in these three sets are 
referred to as S(1-9)A, S(1-9)B, S(1-9)C following the same logic as for S0x sub-samples. The ranges of the total and connected porosities of $\mathrm{S}(1-9) \mathrm{x}$ sub-samples are summarized in Tab.2. The $\varepsilon_{t}$ range of $\mathrm{S}(1-9) \mathrm{C}$ is about two times larger than that of $\mathrm{S}(1-9) \mathrm{A}$ and $\mathrm{S}(1-$ 9 )B sets which indicates a higher degree of spatial variability of geometrical properties for the images treated by the Otsu method. The sub-samples from S(1-9)x confirm a very good connectivity of three image stacks since only S6C and S8C originating from the image stack treated by the Otsu method are not percolating.

\begin{tabular}{|l|c|c|c|}
\hline & $\mathrm{S}(1-9) \mathrm{A}$ & $\mathrm{S}(1-9) \mathrm{B}$ & $\mathrm{S}(1-9) \mathrm{C}$ \\
\hline$\varepsilon_{t}$ & $0.2539-0.3808$ & $0.1099-0.2153$ & $0.0738-0.2858$ \\
$\varepsilon_{c}$ & $0.2442-0.3779$ & $0.1024-0.2142$ & $0.0274-0.2843$ \\
\hline$K_{B B}(x), \mathrm{m}^{2}$ & $8.55 \cdot 10^{-19}-6.54 \cdot 10^{-18}$ & $2.97 \cdot 10^{-20}-1.63 \cdot 10^{-18}$ & $1.78 \cdot 10^{-20}-1.54 \cdot 10^{-17}$ \\
$K_{B B}(y), \mathrm{m}^{2}$ & $9.96 \cdot 10^{-19}-5.25 \cdot 10^{-18}$ & $5.75 \cdot 10^{-20}-7.91 \cdot 10^{-19}$ & $6.95 \cdot 10^{-20}-6.08 \cdot 10^{-18}$ \\
$K_{B B}(y), \mathrm{m}^{2}$ & $1.36 \cdot 10^{-18}-8.48 \cdot 10^{-18}$ & $5.38 \cdot 10^{-20}-1.24 \cdot 10^{-18}$ & $8.91 \cdot 10^{-20}-2.90 \cdot 10^{-18}$ \\
\hline$<K_{B B}(x)>, \mathrm{m}^{2}$ & $2.2807 \cdot 10^{-18}$ & $3.6648 \cdot 10^{-19}$ & $2.7376 \cdot 10^{-18}$ \\
$<K_{B B}(y)>\mathrm{m}^{2}$ & $2.5556 \cdot 10^{-18}$ & $3.0459 \cdot 10^{-19}$ & $1.5531 \cdot 10^{-18}$ \\
$<K_{B B}(z)>, \mathrm{m}^{2}$ & $3.5265 \cdot 10^{-18}$ & $4.3770 \cdot 10^{-19}$ & $8.5057 \cdot 10^{-19}$ \\
\hline
\end{tabular}

Table 2: Variation ranges and mean values of geometrical and transport characteristics for $\mathrm{S}(1-9) \mathrm{x}$ sub-samples.

\subsubsection{Study in continuous flow regime \\ Effect of sample location.}

First, the Stokes flow with the no-slip bounce-back boundary condition (17) is simulated, and the $K_{B B}$ permeability of all the 30 sub-samples along the three coordinate axes was calculated. The obtained values are displayed as function of porosity in Figs.8(b)-(d), and their variation ranges and mean values are summarized in Tab.2. The permeability values of S0x sub-samples can be found in Tab.1. It can be observed that, generally, permeability grows with porosity which is a general trend for clays [41], however, significant variations related to the individual pore space geometry cannot be excluded. The trend is clearly more pronounced for the sub-samples treated by the Otsu method. For $\mathrm{S}(1-9) \mathrm{A}$ and $\mathrm{S}(1-9) \mathrm{B}$ sub-samples, the $K_{B B}(z)$ values are generally higher than $K_{B B}(y)$ and $K_{B B}(x)$. This is more obvious when considering the mean values $\left\langle K_{B B}(x)\right\rangle$, $<K_{B B}(y)>,<K_{B B}(z)>$ which for $\mathrm{S}(1-9)$ A sub-samples follow in the same order as the mean local pore sizes $\left\langle H_{l}^{x}\right\rangle,\left\langle H_{l}^{y}\right\rangle,\left\langle H_{l}^{z}\right\rangle$, thus confirming the existence of a certain spatial anisotropy which is expected for a material of this kind. S(1-9)C sub-samples do not demonstrate the same tendency with $\left\langle K_{B B}(x)>\right.$ being higher then $<K_{B B}(y)>$ and $<K_{B B}(z)>$. The permeability variations are limited to one order of magnitude for S(1-9)A sub-samples, two orders of magnitude for S(1-9)B sub-samples, and three orders of magnitude for S(1-9)C sub-samples, which confirms larger spatial heterogeneity of the samples treated by the erosion and the Otsu method. Generally, the mean permeability of the samples treated by the erosion method is smaller than that of the samples treated by the Otsu method, with $\left\langle K_{B B}>\right.$ of the samples treated by the original Gaboreau et al method being the largest, with the exception of $\left\langle K_{B B}(x)>\right.$. The mean permeability of the $\mathrm{S}(1-9) \mathrm{B}$ samples treated by the erosion method is closer to the experimentally calculated value, with S6B sub-sample being the closest. 


\section{Effect of image resolution.}

In order to estimate the influence of discretization, a refined sample is produced by replacing each voxel of S0A sub-sample with 8 smaller voxels. The permeability of the refined sample $K_{B B}(z)=2.0352 \cdot 10^{-18} \mathrm{~m}^{2}$ is $20.4 \%$ smaller, which indicates that the pore space discretization effects are present but they are reasonably limited.

\subsubsection{Study in slip flow and transitional regime}

Effect of the pore morphology.

To study the Klinkenberg effect, the Kn must be related to physical conditions. For an ideal Boltzmann gas, this can be done by using the following expression of $\mathrm{Kn}$

$$
\mathrm{Kn}=\frac{k_{B} T}{\sqrt{2} \pi d^{2} P_{g} H}
$$

where $k_{B}$ is the Boltzmann constant, $T$ is the temperature, and $d$ is the gas particle kinetic diameter. Thus for $H=53.4750 \mathrm{~nm}$ and the nitrogen gas $\left(d_{N_{2}}=0.364 \cdot 10^{-9}\right.$ $\mathrm{nm}[30]$ ) at $T=293.15 \mathrm{~K}$ and $P_{g}=10^{5} \mathrm{~Pa}$, the Knudsen number can be estimated as $\mathrm{Kn}=1.29$, which corresponds to the transitional flow regime. At the nuclear waste storage conditions, much higher gas pressures can be expected notably due to the gas accumulation. Also, pressures exceeding $P_{g}=10^{6} \mathrm{~Pa}$ are necessary for gas to penetrate the water saturated clay, bringing the estimated Kn down to 0.129 which is still well beyond the continuous flow limits thus justifying the usage of slip-flow boundary condition for the considered illite sample.

For simulations inside a unit cell with spatially periodic boundary conditions and a small external body force, the fluid pressure variations are very small, and, therefore, $P_{g}$ can be considered as constant, and it can be associated with $P_{m}$. On the other hand, $H$ is essentially a local parameter and may vary significantly through the pore-space, whereas, in the presented slip-flow tests, an unique value of $H$ was used to calculate Kn. Therefore, following [54, 35], a local pore size $H_{l}$ associated with each fluid lattice node was introduced for Kn calculation, in order to estimate the resulting variation of $K$ compared to the globally defined $\mathrm{Kn}$. In all the conducted slip flow simulations, the difference between the $K$ values obtained with the globally defined $H_{l}=<H_{l}>$ and with the locally variable $H_{l}$ is very small (less than $1 \%$ ).

The $H_{l}$ is calculated by measuring the distances from the fluid lattice node to the solid surface following the directions of $D 3 Q 27$ discrete velocities. Then, the mean value of these 13 distances is taken as the local pore size $H_{l}$. The $H_{l}$ distribution inside the S0x and S6B sub-samples is displayed in Fig.9(a), while the mean values are summarized in Tab.1. The peak value of S0A corresponds to the fluid lattice nodes with $50<H_{l} \leq 55$ $\mathrm{nm}$ which means that significant part of the pores are quite narrow and poorly discretized, and the mean value is $\left\langle H_{l}\right\rangle=10.6950$ lattice units (or $=53.4750 \mathrm{~nm}$ ). S0B and S6B contain a large amount of even smaller pores which results in their reduced permeability. The $H_{l}$ distribution of S0C sub-sample somehow differs from the others, by having a larger proportion of pores larger than $100 \mathrm{~nm}$, which can be related with different porespace structure. Comparing the mean local pore sizes $\left\langle H_{l}\right\rangle_{x},\left\langle H_{l}\right\rangle_{y}$, and $\left\langle H_{l}\right\rangle_{z}$ (see Tab.1) measured only along the principal axes directions, it is clear that the pores are preferentially aligned along the $z$-axis for all the the sub-samples followed by $\left\langle H_{l}\right\rangle_{y}$, with $\left.<H_{l}\right\rangle_{x}$ being the smallest. 
A significant difference in pore-space structure between the sub-samples treated by the Otsu method and those treated by the Gaboreau et al [19] and the erosion method can be confirmed by considering a characteristic pore size $l_{z}$, which is a $2 D$ parameter calculated for each image as

$$
l_{z}=\frac{S_{\text {pore }}}{l_{\text {pore }}}
$$

where $S_{\text {pore }}$ is the area of the pore, and $l_{\text {pore }}$ is the length of the pore border. The $l_{z}$ of the S0x and S6B sub-samples is displayed in Fig.9(b). The S0A and S0B subsamples are quite similar with difference mainly due to different porosities. The S6B sub-sample extracted from a different location presents similar characteristics with S0B. On the other hand, S0C sub-sample is quite different with $l_{z}$ presenting significantly larger variations and absolute values, while the S0C porosity is smaller than that of other presented sub-samples. This indicates that S0C pores have more bulbous shape compared to the narrow slit-like pores of the sub-samples treated by the Gaboreau et al [19] and the erosion method thus influencing slip flow local velocities.

\section{The Klinkenberg effect.}

To study the Klinkenberg effect, the fluid flow with the slip boundary condition (18) was simulated inside the S0x and S0B sub-samples along the $z$ - axis. Kn is ranging from $10^{-4}$ to 0.4 . The Kn values can be related to the mean gas pressure inside the pores $P_{m}$ by means of (33) supposing the nitrogen gas at $T=293.15 \mathrm{~K}$. As it was mentioned above, the simulations conducted with $H=<H_{l}>$ everywhere and simulations performed with site by site calculated $H_{l}$ provide the permeability results within $1 \%$, therefore, $H$ in (33) can be replaced with $\left\langle H_{l}>\right.$.

The influence of the Knudsen number on fluid velocity can be observed by considering the distribution of the scaled velocity norm $\frac{\|\mathbf{u}\|}{\|\mathbf{u}\|_{\max }}$ inside the pore space of S0A sub-sample as displayed in Fig.10. For relatively small Kn = 0.01, Fig.10(a) exhibits preferential pathways where the most of the flow is concentrated. As expected, the velocity is higher in the largest pores. Conversely, for large $\mathrm{Kn}=0.4$, gas velocity increases in the narrow passages due to slippage effect and fluid flow is more uniformly distributed as can be observed in Fig.10(b). It follows, that this mechanism contributes to regulate the overall flow inside the pore space as Kn grows.

The calculated permeability for various Kn values is displayed in Fig.6(b) where it is plotted versus the inverse of $P_{m}$. The obtained $K$ values are very well aligned and can be linearly fitted by (2) with $K_{0}$ and $b$ summarized in Tab.1. As noted in Sec.3.1, the $K_{0}$ obtained from the linear fit is smaller than $K_{B B}(z)$ obtained with the bounce-back boundary condition.

The Klinkenberg factor $b$ varies significantly from sample to sample depending on the pore-space geometry. The data are highly scattered. Therefore, multiple studies were conducted in effort to correlate the $b$ factor with other transport properties, mainly $K_{0}$. Usually, the correlation takes the form of the power law. Thus the $b$ factor for oil-field cores with $K_{0}$ ranging from $10^{-17} \mathrm{~m}^{2}$ to $10^{-12} \mathrm{~m}^{2}$ was correlated by [29] with

$$
b=0.11 K_{0}^{-0.39}
$$

where $K_{0}$ is expressed in $\mathrm{m}^{2}$ unit, and $b$ is given in Pa unit. Later, the $b$ factor for 100 core plugs with $K_{0}$ lying in the same range (from $10^{-17} \mathrm{~m}^{2}$ to $10^{-12} \mathrm{~m}^{2}$ ) was fitted by 
[31] with

$$
b=6.9 K_{0}^{-0.36}
$$

where $K_{0}$ is expressed in $\mathrm{mD}$ unit, and $b$ is given in psi unit. In [32], the $b$ factor for low-permeable sands with $K_{0}$ varying between $10^{-19} \mathrm{~m}^{2}$ and $10^{-14} \mathrm{~m}^{2}$ was estimated as

$$
b=0.86 K_{0}^{-0.33}
$$

with $K_{0}$ and $b$ expressed in $\mathrm{m}^{2}$ and Pa unit, respectively. The study of the metagraywacke plug samples with $K_{0}$ ranging from $2 \times 10^{-22} \mathrm{~m}^{2}$ to $4 \times 10^{-20} \mathrm{~m}^{2}$ has delivered in [45] the $b$ factor values lying between $1 \mathrm{MPa}$ and $20 \mathrm{MPa}$. Recently, the $b$ factor was estimated by [50] based on the data for 30 Taiwan sedimentary rocks (330 data points in total) with $K_{0}$ ranged from $10^{-20} \mathrm{~m}^{2}$ to $10^{-14} \mathrm{~m}^{2}$ delivering the following estimation

$$
b=(0.15 \pm 0.06) K_{0}^{-0.37 \pm 0.038}
$$

where $K_{0}$ and $b$ are given in $\mathrm{m}^{2}$ and $\mathrm{Pa}$ unit, respectively. Finally, using the fluid flow between the parallel plates as a starting point, an analytical expression for $b$ factor was obtained in [52] for granular and foam models

$$
b=12 P_{m} \frac{2-\sigma}{\sigma} \mathrm{Kn}
$$

where $\sigma$ is the tangential momentum accommodation coefficient.

For further analysis, these correlations together with the experimentally obtained $b$ factor of the compacted illite powder sample, the numerically calculated $b$ factor of the S0x and S6B sub-samples, and the $b$ factor experimentally measured by Boulin [9] for a clay sample are summarized in Fig.11. It can be noted, that two points corresponding to the experimentally measured $b$ factor values are located in close proximity to each other although the samples are of different origin, which is in a good agreement with general correlation established between the $b$ factor and absolute permeability $K_{0}$. It is remarkable that the $b$ factors calculated for the sub-samples treated by the Gaboreau et al [19] and the erosion method are in a very good agreement with other correlation trends in spite of the small number of points. The $K_{0}$ value of S6B sub-sample is the closest to the measured experimentally $K_{0}$ value of the illite sample, and its $b$ factor is $33.45 \%$ smaller than that of the illite sample, which can be considered as a good numerical estimation. On the other hand, the $b$ factor of S0C sample treated by the Otsu method falls out of the general trend followed by S0A, S0B, and S6B sub-samples, which can be explained by the different pore space structure (see Fig.9(b)) with domination of bulbous shaped pores. Using (33) along with $H=\left\langle H_{l}\right\rangle$, the Klinkenberg factor of the selected subsamples can be also estimated with (39), however, resulting estimations will not follow the power law trend.

\section{Conclusions}

In this study, we address the question of the LBM applicability to nanoporous materials using the 3D TRT-LBM model combined with the diffuse reflection boundary condition to describe the slip flow regime. Simulations conducted on several test cases highlight the accuracy of the method provided that an accurate representation of the 
pore-scale geometry is available. In constrast with the continuous flow regime, as predicted by the bounce-back boundary condition, slip flow simulations are highly dependent on the morphological features such as pore surface roughness. Results indicate a decrease of the permeability for rough surfaces and this behavior is consistent with the model of apparent permeability derived by Darabi et al [14] using the Maxwell theory. In a second part, the LBM is applied to numerically estimate the transport properties of illite from the digital images treated by three different segmentation techniques. Also, these properties are measured experimentally for the illite sample recreated from the same basic material.

The obtained results demonstrate that the LBM is a versatile method which can be successfully applied to simulation of slip and no-slip flow inside the pore space, thus permitting the calculation of the absolute permeability as well as the estimation of the Klinkenberg factor. Reliable results can be obtained even for poorly discretized pore space with relatively small number of fluid lattice nodes spanning the pore. This analysis supports LBM as a valuable tool for understanding and predicting the slip flow effects at nanometer pore-scale. However, two significant concerns should be kept in mind when applying LBM to nano-porous rock permeability calculations:

First, choice of the segmentation method applied to the digital images appears to have a significant influence on the numerical results. The absolute permeability values calculated for the same sub-sample treated by different segmentation methods can differ by three orders of magnitude. The variability of absolute permeability due to the location of the sub-sample inside the original image is limited to one order of magnitude for the sub-samples treated by the Gaboreau et al [19] method, two orders of magnitude for the sub-samples treated by the erosion method, and three orders of magnitude for the sub-samples treated by the Otsu method. Segmentation methods may also modify the shape of the pores which can influence significantly the calculated Klinkenberg factor. If the pore space structure is not strongly modified by segmentation method, the calculated $b$ factor values follow well the existing empirical trends and a good estimation of $b$ can be obtained from a sub-sample with $K_{0}$ close to the experimental value.

Second, direct LBM calculations at the VER size which is crucial for reliable permeability assessment can be particularly difficult to achieve for nano-porous rocks due to large pore size distribution. In the present study, we circumvent this computational size issue by performing a statistical analysis on several sub-samples so that the mean and variance of the sample permeability can be calculated.

\section{Acknowledgments}

The financial support from NEEDS MIPOR is gratefully acknowledged. This work was also partially funded by the ICEEL Carnot Institute (grant MultiEC-HYDRO). We are thankful to Stephane Gaboreau who provided the digital images of the compacted illite used in this article. 


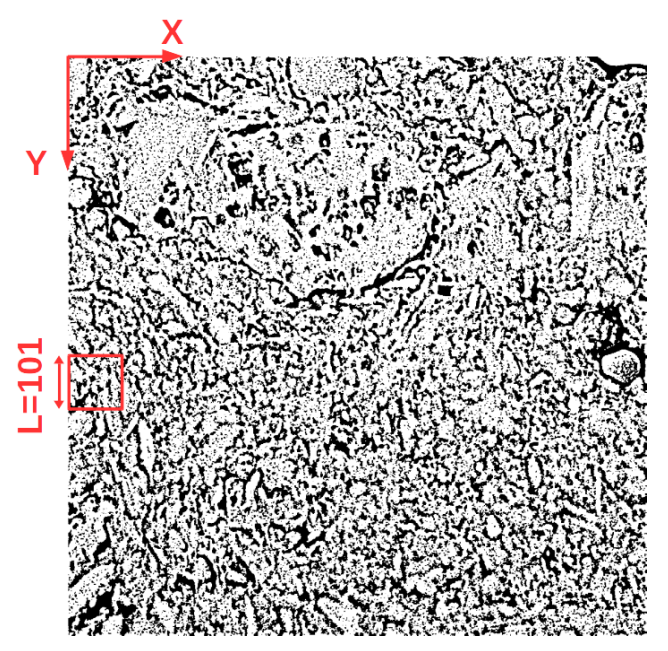

(a)

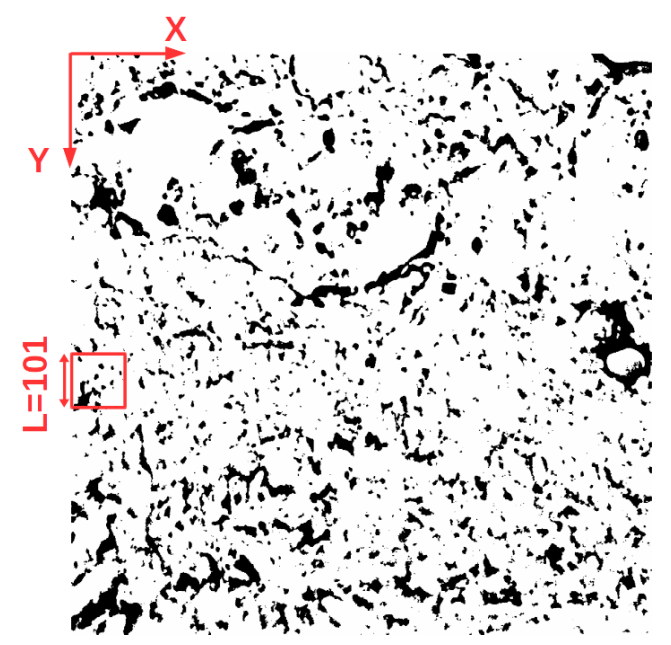

(c)

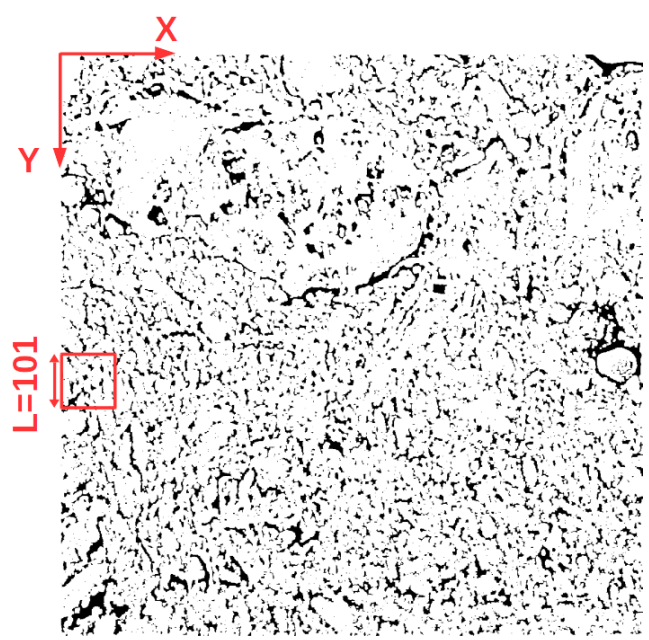

(b)

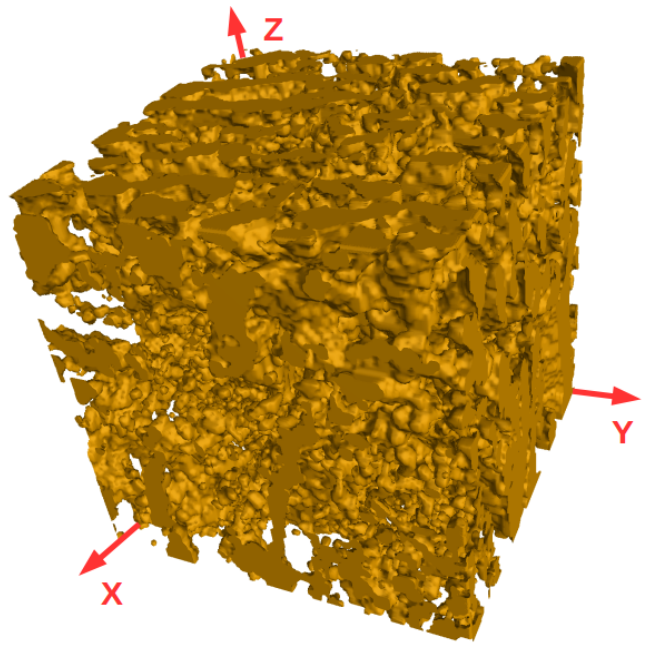

(d)

Figure 7: The first image $(5480 \times 5475 \mathrm{~nm})$ in a stack of the illite sample images treated by (a) the Gaboreau et al advanced method [19]; (b) the erosion method; (c) the Otsu method. The pores are black and the solid matrix is white. The red rectangular $(\mathrm{L}=505 \mathrm{~nm})$ indicates the image size and the location of S0x sub-samples. (d) the 3D view of S0A $(505 \times 505 \times 510 \mathrm{~nm})$ sub-sample pore space. 


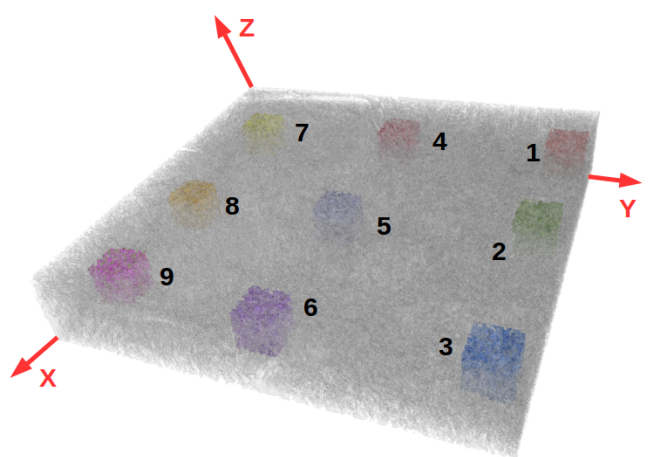

(a)

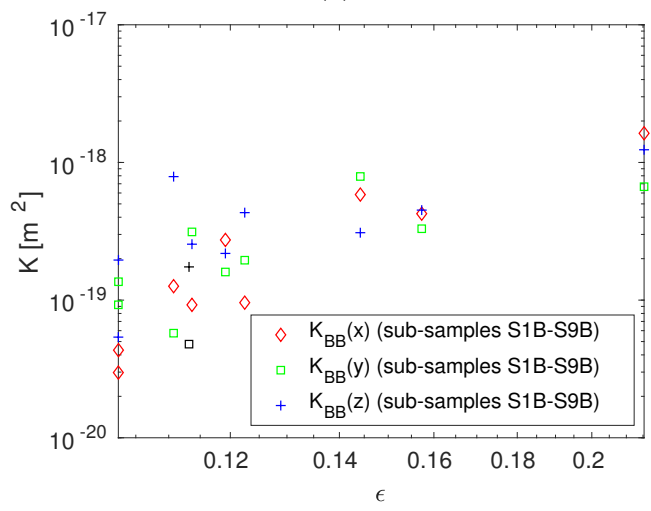

(c)

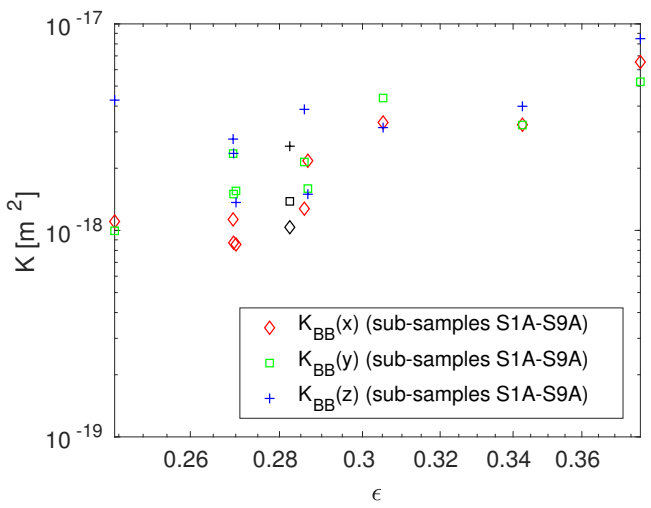

(b)

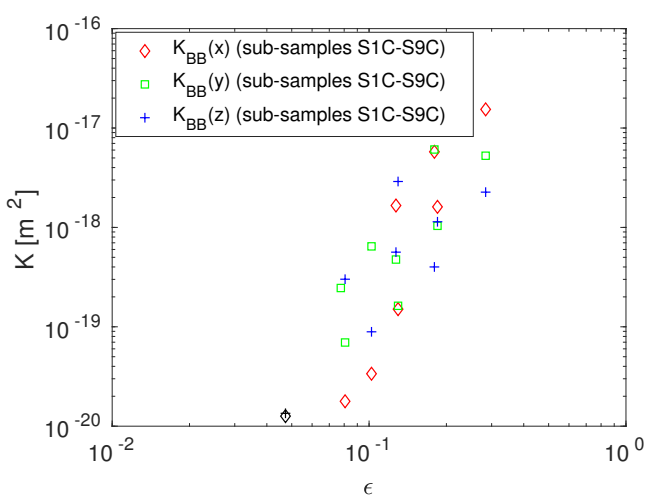

(d)

Figure 8: Locations (a) of the additionally extracted $\mathrm{S}(1-9) \mathrm{x}$ sub-samples. The $K_{B B}$ permeability calculated along three coordinate axes for S(1-9)A (b), S(1-9)B (c), S(1-9)C (d) (colored), and for S0x (black) sub-samples as function of porosity. 


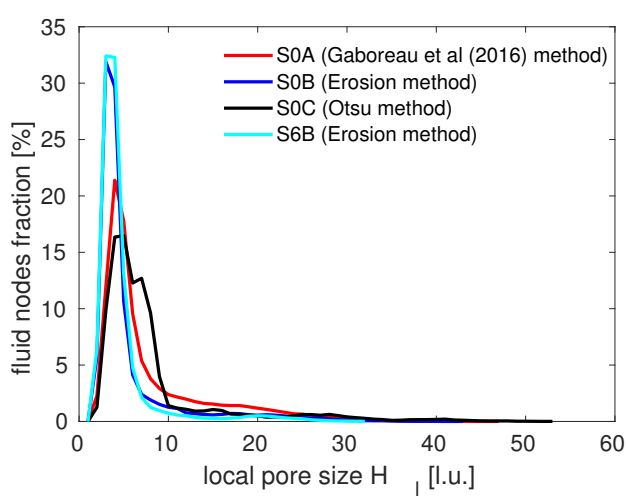

(a)

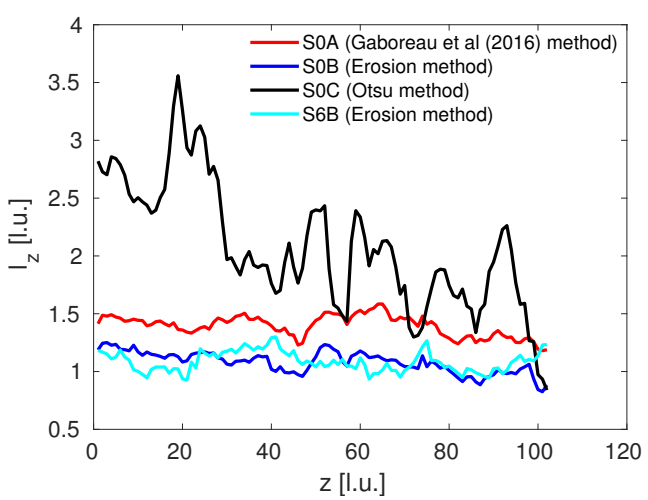

(b)

Figure 9: (a) The local pore size $H_{l}$ distribution inside the illite sub-samples. (b) Characteristic pore size $l_{z}$ of the extracted illite sub-samples.

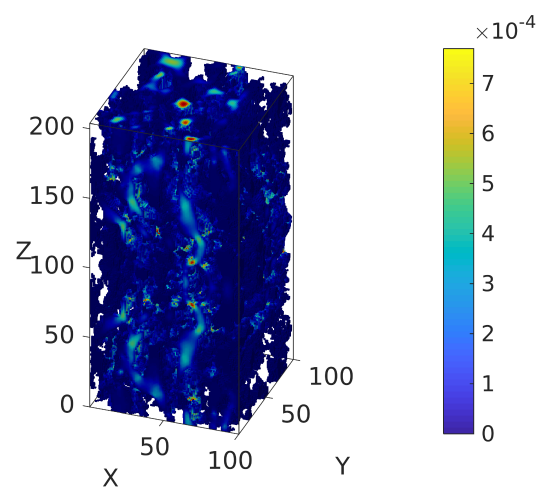

(a)

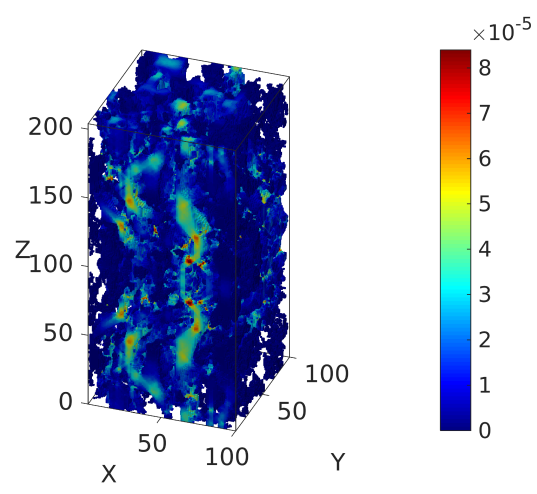

(b)

Figure 10: Scaled velocity norm $\frac{\|\mathbf{u}\|}{\|\mathbf{u}\| \text { max }}$ distribution inside the pore space of S0A sub-sample for (a) $\mathrm{Kn}=0.01$ and (b) $\mathrm{Kn}=0.4$. 


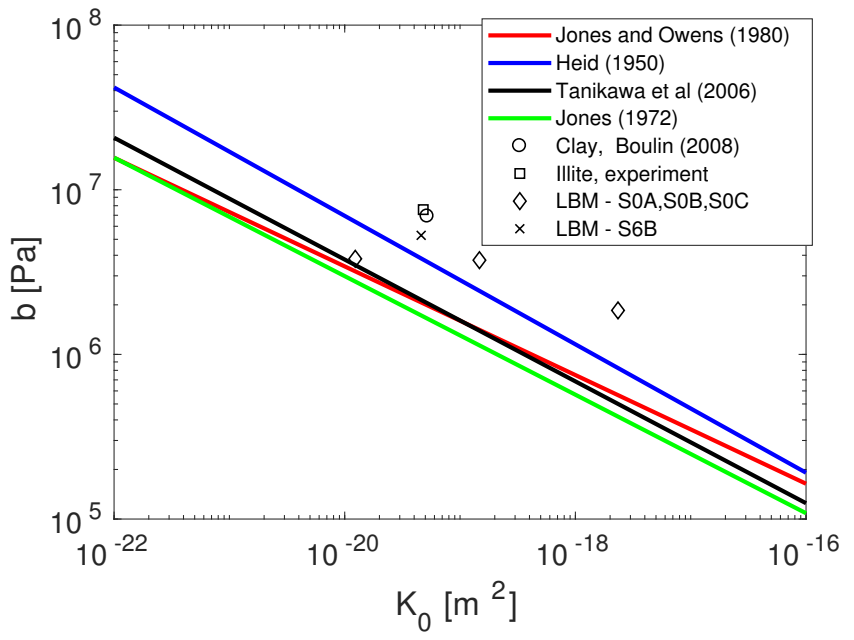

Figure 11: Klinkenberg factor $b$ as function of absolute permeability $K_{0}$. 
[1] F.O. Alpak, F. Gray, N. Saxena, J. Dietderich, R. Hofmann, S. Berg. A distributed parallel multiplerelaxation-time lattice Boltzmann method on general-purpose graphics processing units for the rapid and scalable computation of absolute permeability from high-resolution 3D micro-CT images. Comput. Geosci. 22, 815-832, (2018).

[2] ANDRA Dossier 2005 Argile. Synthesis. Evaluation of the feasibility of a geological repository in an argillaceous formation. ANDRA Report Series, 782 p., ISBN 2-916162-00-3, (2005).

[3] S. Ansumali and I.V. Karlin. Kinetic boundary conditions in the lattice Boltzmann method. Phys. Rev. E 66, 026311 (2002).

[4] F. Bardelli, C. Mondelli, M. Didier, J.G. Vitillo, D. R. Cavicchia, J.-C. Robinet, L. Leone, L. Charlet. Hydrogen uptake and diffusion in Callovo-Oxfordian clay rock nuclear waste disposal technology. Applied Geochemistry, 49, 168-177, (2014).

[5] S. Ben Hadj Hassine, M. Dymitrowska, V. Pot, A. Genty. Gas Migration in Highly Water-Saturated Opalinus Clay Microfractures Using a Two-Phase TRT LBM. Transp. Porous Med. 116, 975-1003, (2017).

[6] M. Benioug, F. Golfier, A.J. Tinet, M.A. Buès and C. Oltéan. Numerical efficiency assessment of IB-LB method for 3D pore-scale modeling of flow and transport. Transp. Porous Med., 109, 1-23 (2015).

[7] M. Benioug, F. Golfier, C. Oltéan, M.A. Buès, T. Bahar and J. Cuny. An immersed-boundary lattice Boltzmann model of biofilm growth in porous media. Adv. Water Resour., 107, 65-82 (2017).

[8] P. L. Bhatnagar, E. P. Gross and M. Krook. A model for collision processes in Gases. I. Small amplitude processes in charged and neutral one-component systems. Phys. Rev. 94, 511 (1954).

[9] P. Boulin. Expérimentation et Modélisation du Transfert d'hydrogène à travers des argiles de centre de stockage de déchets radioactifs. Ph.D. thesis. Institut National Polytechnique de Grenoble, (2008).

[10] Z. H. Chai, Z. L. Guo, L. Zheng, and B. C. Shi. Lattice Boltzmann simulation of surface roughness effect on gaseous flow in a microchannel. J. Appl. Phys. 104, 014902 (2008).

[11] Z. Chai, B. Shi, Z. Guo and J. Lu. Gas Flow Through Square Arrays of Circular Cylinders with Klinkenberg Effect: A Lattice Boltzmann Study, Commun. Comput. Phys. 8, No. 5, 1052-1073, (2010).

[12] B. Chun and A.J.C. Ladd. Interpolated boundary condition for lattice Boltzmann simulations of flows in narrow gaps. Phys Rev E 75, 066705 (2007).

[13] R. Cuss, J. Harrington, R. Giot, C. Auvray. Experimental observations of mechanical dilatation at the onset of gas flow in Callovo-Oxfordian claystone. Clays in Natural and Engineered Barriers for Radioactive Waste Confinement, 400, 507-519, (2014).

[14] H. Darabi, A. Ettehadtavakko, F. Javadpour and K. Sepehnoori. Gas flow in ultra-tight shale strata. J. Fluid Mech., 710, 641-658 (2012).

[15] C. Davy, S. M'Jahad, F. Skoczylas, J. Talandier. Migration de gaz au travers de l'argilite de Bure: mise en evidence d'un passage discontinu. Rencontres AUGC (2013).

[16] C.A. Davy, P.M. Adler. Three-scale analysis of the permeability of a natural shale. Phys. Rev. E 96, 063116, (2017)

[17] D. d'Humières, I. Ginzburg, M. Krafczyk, P. Lallemand and Li-Shi Luo. Multiple-relaxation-time lattice Boltzmann models in three dimensions. Phil. Trans. R. Soc. Lond. A 360, 437 (2002).

[18] M. Dymitrowska, A. Pazdniakou and P.M. Adler. Two-phase flow pore-size simulations in Opalinus clay by the lattice Boltzmann method. Geological Society, London, Special Publications, 400, 195206 (2014)

[19] S. Gaboreau, J.-C. Robinet, D. Prêt, Optimization of pore-network characterization of a compacted clay material by TEM and FIB/SEM imaging, Microporous and Mesoporous Materials 224, 116$128,(2016)$.

[20] I. Ginzbourg and P.M. Adler. Boundary flow condition analysis for the three-dimensional lattice Boltzmann model. J. Phys. II France 4, 191 (1994).

[21] I. Ginzburg and D. d'Humières. Multireflection boundary conditions for lattice Boltzmann models. Phys. Rev. E 68, 066614 (2003).

[22] Z. Guo, T.S. Zhao and Y. Shi. Physical symmetry, spatial accuracy, and relaxation time of the lattice Boltzmann equation for microgas flows. J. Appl. Phys., 99, 074903 (2006).

[23] Z. Guo and C. Zheng. Analysis of lattice Boltzmann equation for microscale gas flows: Relaxation times, boundary conditions and the Knudsen layer. Int. J. Comput. Fluid Dyn. 22, 7, 465-473, (2008).

[24] Z. Guo, C. Shu, Lattice Boltzmann Method and its Applications in Engineering. Advances in Computational Fluid Dynamics, 3, (2013). 
[25] N.G. Hadjiconstantinou. Comment on Cercignani's second-order slip coefficient. Physics of Fluids 15, $2352(2003)$

[26] J.F. Harrington, R. de la Vaissiere, D.J. Noy, R.J. Cuss, J. Talandier. Gas flow in Callovo-Oxfordian claystone $(\mathrm{COx})$ : results from laboratory and field-scale measurements. Mineralogical Magazine, 76(8), 3303-3318, (2012).

[27] X. He and L.-S. Luo. A priori derivation of the lattice Boltzmann equation. Phys. Rev. E 55, 6333 (1997).

[28] X. He and L.-S. Luo. Theory of the lattice Boltzmann method: From the Boltzmann equation to the lattice Boltzmann equation. Phys. Rev. E 56, 6811 (1997).

[29] J.G. Heid, J.J. Mcmahon, R.F. Nielsen and S.T. Yuster. Study of the permeability of rocks to homogeneous fluids, American Petroleum Institute, Drilling and Productions practices, New York 230-244, (1950)

[30] A. F. Ismail, K.C. Khulbe, T. Matsuura. Gas Separation Membranes. Polymeric and Inorganic, Springer, (2015)

[31] S.C. Jones, A rapid accurate unsteady-state Klinkenberg permeameter, Soc. Pet. Engr. J., 3535, 383-397, (1972).

[32] F.O. Jones and W.W. Owens. A laboratory study of low permeability gas sands. J. Pet. Technol., 32, 1631-1640, (1980).

[33] L. J. Klinkenberg, The permeability of porous media to liquid and gases, American Petroleum Institute, Drilling and Productions practices, New York, (1941).

[34] A. J. C. Ladd and R. Verberg. Lattice-Boltzmann simulations of particle-fluid suspensions. J. Stat. Phys. 104, 1191 (2001)

[35] K.J. Lange, P.-C. Sui, and N. Djilali. Pore Scale Simulation of Transport and Electrochemical Reactions in Reconstructed PEMFC Catalyst Layers. Journal of The Electrochemical Society, 157 (10), B1434-B1442 (2010).

[36] Li-Shi Luo. Theory of the lattice Boltzmann method: Lattice Boltzmann models for nonideal gases. Phys. Rev. E 62, 4982 (2000).

[37] P. Marschall, S. Horseman, T.Gimmi. Characterisation of Gas Transport Properties of the Opalinus Clay, a Potential Host Rock Formation for Radioactive Waste Disposal. Oil \& Gas Science and Technology - Rev. IFP, 60, 121-139, (2005).

[38] G.R. McNamara and G. Zanetti. Use of the Boltzmann equation to simulate lattice-gas automata. Phys. Rev. Lett. 61, 2332 (1988).

[39] C. McPhee, J. Reed, I. Zubizarreta. Core analysis: a best practice guide. Developments in Petroleum Science, 64, 852, (2015).

[40] Mitra, S.K. and Chakraborty, S. Microfluidics and Nanofluidics Handbook, Two Volume Set. CRC Press, (2011).

[41] C.E. Neuzil. How permeable are clays and shales? Water Resour. Res. 30, 145-150, (1994).

[42] C. Pan, Li-Shi Luo, Cass T. Miller. An evaluation of lattice Boltzmann schemes for porous medium flow simulation. Computers \& Fluids 35, 898 (2006).

[43] A. Pazdniakou and P.M. Adler. Dynamic permeability of porous media by the lattice Boltzmann method. Adv. Water Resour., 62, 292-302 (2013).

[44] A. Pazdniakou and M. Dymitrowska. Migration of gas in water-saturated clays by coupled hydraulicmechanical model. Geofluids, 2018, ID 6873298, 25 pages (2018).

[45] P. Persoff, J.B. Hulen. Hydrologic characterization of reservoir metagraywacke from shallow and deep levels of The Geysers vapor-dominated geothermal system, California, USA. Geothermics, 30 , 169-192, (2001).

[46] Y. Song, C.A. Davy, D. Troadec, A.-M. Blanchenet, F. Skoczylas, J. Talandier, J.C. Robinet. Multi-scale pore structure of COx claystone: Towards the prediction of fluid transport. Marine and Petroleum Geology, 65, 63-82, (2015).

[47] S. Succi. Mesoscopic modeling of slop motion at fluid-solid interfaces with heterogeneous catalysis Phys. Rev. Lett. 89, 064502 (2002).

[48] K. Suga. Corrigendum: Lattice Boltzmann methods for complex micro flows: applicability and limitations for practical applications. Fluid Dyn. Res. 45, 059501 (2013).

[49] G.H. Tang, W.Q. Tao, and Y. L. He. Lattice Boltzmann method for gaseous microflows using kinetic theory boundary conditions, Phys. Fluids, 17, 058101 (2005).

[50] W. Tanikawa, T. Shimamoto. Klinkenberg effect for gas permeability and its comparison to water permeability for porous sedimentary rocks. Hydrology and Earth System Sciences Discussions, European Geosciences Union, 3 (4), 1315-1338 (2006).

[51] S. Tao and Z. Guo. Boundary condition for lattice Boltzmann modeling of microscale gas flows with 
curved walls in the slip regime. Phys. Rev. E 91, 043305 (2015).

[52] S. Woudberg, J.P. Du Plessis. Predicting the Permeability of Very Low Porosity Sandstones. Transp. Porous Med., 73, 39-55 (2008)

[53] D. Zhang, R. Zhang, S. Chen, and W.E. Soll. Pore scale study of flow in porous media: Scale dependency, REV, and statistical REV. Geophysical Research Letters vol. 27, no. 8, 1195-1198, (2000).

[54] J. Zhao, J. Yao, M. Zhang, L. Zhang, Y. Yang, H. Sun, S. An and A. Li. Study of Gas Flow Characteristics in Tight Porous Media with a Microscale Lattice Boltzmann Model. Sci. Rep., 6, 32393 (2016) 